A. Pramesh Rao, G. Swarup and Gopal-Krishna, eds.

\title{
VLA Images of two Extended Radio Galaxies
}

\author{
W. Junor \\ Institute for Astrophysics, Univ. of New Mexico, Albuquerque, NM, USA \\ F. Mantovani, R. Morganti and L. Padrielli \\ Istituto di Radioastronomia del CNR, Bologna, Italy
}

\begin{abstract}
There is some evidence from earlier studies that the two sources $0235-197$ and $1203+043$ exhibit low frequency $(<1 \mathrm{GHz})$ variability. This work shows that both sources have linear polarizations, if any, below the detection limits at $320 \mathrm{MHz}$, so we cannot explain the variability as being due to instrumental polarization effects as has been suggested for $3 \mathrm{C} 159$. Refractive scintillation may be the cause of the variability in $0235-197$. The radio source $1203+043$ lacks any bright compact component thereby ruling out a refractive scintillation mechanism for its variability. Consequently, it is possible that claims of variability in this source are spurious. However, the $320 \mathrm{MHz}$ VLA observations show that $1203+043$ has an ' $\mathrm{X}$ '-shaped radio structure.
\end{abstract}

\section{Introduction}

As part of our investigation of steep-spectrum $\left(\alpha>0.5, \mathrm{~S} \propto \nu^{-\alpha}\right)$, low-frequencyvariable (LFV; $\nu<1 \mathrm{GHz}$ ) sources (Mantovani et al. 1992), we have made a series of VLA images with sub-arcsecond resolutions of the two sources 0235-197 and $1203+043$. The aim was to detect the high-brightness components required by the refractive scintillation model for low-frequency variability (Rickett 1986). Alternatively, the variability, detected with linearly polarized antennas, might be explained as an instrumental effect. The plane of polarization of the emission could be rotated by changes in ionospheric Faraday rotation relative to the antenna's dipole as found by Cerchiara et al. (1994) for 3C159. In order to test if ionospheric Faraday rotation is the cause of the apparent variability of 0235-197 and 1203+043 we have investigated the linear polarizations of these sources at $320 \mathrm{MHz}$ with the VLA in the 'A' configuration and with the already available $5 \mathrm{GHz}$ VLA C-array data. Both sources were also observed in the $\mathrm{X}$ $(8.4 \mathrm{GHz})$ and $\mathrm{U}(15 \mathrm{GHz})$ bands. These observations allowed high resolution images of the 'hot spot' regions to be made. The images were combined with available, high-resolution, $\mathrm{C}$ band observations to produce rotation measures (RMs) for the outer parts of the sources. The images obtained at $320 \mathrm{MHz}$ for the two sources are shown in Figure 1. 

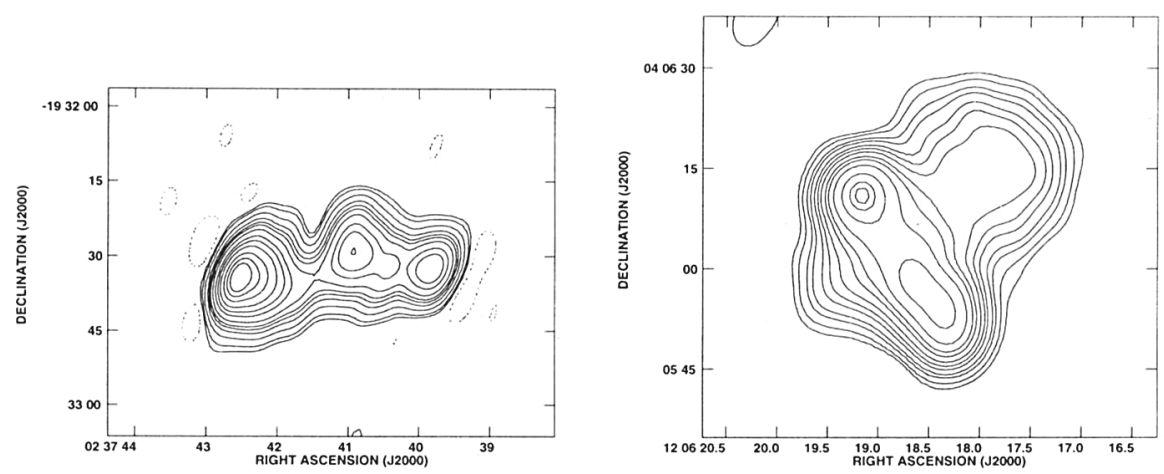

Figure 1. VLA image of $0235-197$ and $1203+043$ at $320 \mathrm{MHz}$. Contours are at $-5,5,10,20,50,100,150,200,300,500,700,1000,1500$, $2000,2500 \mathrm{mJy}^{\text {beam }}{ }^{-1}$. The peak flux densities are $3430 \mathrm{mJy} \mathrm{beam}^{-1}$ and $800 \mathrm{mJy}^{\text {beam }}{ }^{-1}$ respectively.

\section{Conclusions}

We have conducted a program of multi-wavelength VLA observations of the suspected low frequency variable sources $0235-197$ and $1203+043$. Since $0235-197$ is not polarized at $320 \mathrm{MHz}$, its variability cannot be accounted for by instrumental polarization effects as in the case of 3C159. 0235-197 may contain a low frequency component sufficiently compact and bright as required by the refractive scintillation model for low frequency variability. Our observations have insufficient resolution to test this suggestion; low frequency VLBI observations are required for this purpose. However, this component would have to have extremely unusual properties among hot spots in radio sources.

In our high frequency images of $1203+043$ we have identified the core of the radio source; its location indicates that the source has a large apparent asymmetry. At $320 \mathrm{MHz}$, this source shows no polarization. However, it does have an additional, steep-spectrum component at this frequency; this previouslyundetected component lies perpendicular to the main axis and predominantly to one side. However, the overall morphology of $1203+043$ at low frequencies seems similar to that of the ' $\mathrm{X}$ '-shaped sources like NGC326. From its morphology and component sizes, we conclude that $1203+043$ is likely not variable at low frequencies and that its inclusion in such catalogs is spurious.

\section{References}

Cerchiara, P.L. et al., 1994, MNRAS, 267, 247

Mantovani, F. et al., 1992, MNRAS, 257, 353

Rickett B.J., 1986, ApJ, 307, 564 